\title{
Enraizamiento de miniestacas de Guazuma crinita M. utilizando diferentes invernaderos, sustratos y aditivos
}

\author{
Rooting of minicuttings of Guazuma crinita M. using different \\ greenhouses, substrates and additives
}

\author{
Yanisse Basauri Torres ${ }^{1}$, Wilson Francisco Guerra Arévalo², Guillermo Eduardo \\ Gorbitz Dupuy ${ }^{3}$, Ignacio Lombardi Indacochea ${ }^{4}$, Héctor Guerra Arévalo ${ }^{2}$, Eduardo \\ Medeiros de Oliveira ${ }^{5}$, Joao Luiz Lopes Monteiro Neto ${ }^{5}$, Dennis del Castillo Torres $^{2}$ y \\ Krystel Clarissa Rojas Mego ${ }^{2}$ y Carlos Abanto-Rodríguez ${ }^{2}$
}

\begin{abstract}
Resumen
El objetivo en este trabajo fue determinar el mejor invernadero, sustrato y aditivo para el enraizamiento de miniestacas de G. crinita M. para ello fue utilizado material apical de $5 \mathrm{~cm}$ de longitud, $4 \mathrm{~mm}$ de diámetro y $2 / 3$ de área foliar (20-30 $\left.\mathrm{cm}^{2}\right)$, provenientes de plantas de G. crinita M. Después de 20 días, fue determinado entre 93,3 y $100 \%$ de enraizamiento, $100 \%$ de sobrevivencia y $100 \%$ de brotes en las miniestacas instaladas en los invernaderos policarbonato y malla raschel ${ }^{\circledR}$ asociados con los sustratos y aditivos arena fina-sanix ${ }^{\circledR}$ y jiffy ${ }^{\circledR}$-rapid root $^{\circledR}$. También fue determinado 18,9 raíces en la combinación de policarbonato-arena fina-rapid root ${ }^{\circledR}$. En malla raschel ${ }^{\circledR}$ y policarbonato con jiffy ${ }^{\circledR}$-rapid root ${ }^{\circledR}$ y rapid $\operatorname{root}^{\circledR}$-sanix ${ }^{\circledR}$ fue determinado 14,5 y 12,6 raíces, respectivamente. En condiciones de policarbonato-arena fina fue constatado $46,1 \mathrm{~cm}$ y en jiffy ${ }^{\circledR}$ con malla raschel ${ }^{\circledR}$ y policarbonato las raíces presentaron 41,4 y $35,3 \mathrm{~cm}$ de longitud, respectivamente. Los invernaderos construidos de policarbonato y de malla raschel ${ }^{\circledR}$ asociados con los sustratos arena fina y jiffy ${ }^{\circledR}$ más los aditivos enraizantes rapid root $^{\circledR}$ y sani ${ }^{\circledR}$ fueron las mejores condiciones para el enraizamiento de miniestacas de G. crinita M.
\end{abstract}

Palabras-clave: Bolaina blanca. Propagación vegetativa. Hormona AIB. Arena fina. Jiffy pellet ${ }^{\circledR}$

\begin{abstract}
The objective in this work was to determine the best greenhouse, substrate and additive for the rooting of minicuttings of $G$. crinita M. For this purpose, apical material of $5 \mathrm{~cm}$ in length, $4 \mathrm{~mm}$ in diameter and $2 / 3$ of leaf area was used $\left(20-30 \mathrm{~cm}^{2}\right)$, from plants of G. crinita M. After 20 days, it was determined between 93.3 and $100 \%$ of rooting, $100 \%$ of survival and $100 \%$ of shoots in the minicuttings installed in the associated polycarbonate and raschel ${ }^{\circledR}$ greenhouses with substrates and sand-sanix ${ }^{\circledR}$ and jiffy ${ }^{\circledR}$-rapid root $^{\circledR}$ additives. It was also determined 18.9 roots in the combination of polycarbonate-fine sand-rapid root $^{\circledR}$. In raschel ${ }^{\circledR}$ and polycarbonate mesh with jiffy ${ }^{\circledR}$-rapid root $^{\circledR}$ and rapid root $^{\circledR}$-sanix ${ }^{\circledR}, 14.5$ and 12.6 roots were determined, respectively. In conditions of polycarbonate-fine sand it was found $46.1 \mathrm{~cm}$ and in jiffy ${ }^{\circledR}$ with raschel ${ }^{\circledR}$ mesh and polycarbonate the roots presented 41.4 and $35.3 \mathrm{~cm}$ in length, respectively. The greenhouses constructed of polycarbonate and raschel ${ }^{\circledR}$ mesh associated with the fine sand and jiffy ${ }^{\circledR}$ substrates plus the rapid root ${ }^{\circledR}$ and sani ${ }^{\circledR}$ rooting additives were the best conditions for the rooting of $G$. crinita $\mathrm{M}$.
\end{abstract}

Keywords: Bolaina Blanca. Vegetative propagation. AIB hormone. Fine sand. Jiffy pellet ${ }^{\circledR}$

\footnotetext{
1. University of Eastern Finland. Kuopio, Finlandia.

2. IIAP - Instituto de Investigaciones de la Amazonía Peruana. Iquitos, Perú.

3. Dipteryx Pucallpa. Ucayali, Perú.

4. UNALM - Universidad Nacional Agraria la Molina. Molina, Perú.

5. UFRR - Universidade Federal de Roraima. Boa Vista, RR, Brasil.

* autor correspondiente: carforestal24@gmail.com
}

Sci. For., Piracicaba, v. 47, n. 124, p. 632-643, dez. 2019 DOI: doi.org/10.18671/scifor.v47n124.05 


\section{INTRODUCCIÓN}

El Perú, según el SINIA-MINAM (2016) posee 68733265 millones de hectáreas de bosques amazónicos, en virtud de eso, ocupa el segundo lugar con mayor cobertura forestal después de Brasil en América Latina y el noveno en el mundo. No obstante, Ríos (2014), cita que, a pesar del enorme potencial forestal, hasta el año 2012 existió una oferta comercial negativa con déficit de \$ 676 millones de USD. En ese sentido, para incrementar el volumen de madera comercial se tiene que buscar estrategias y alternativas para atender la demanda creciente en el mercado local e internacional.

Dentro de estas alternativas, el manejo silvicultural de los rodales naturales y el establecimiento de plantaciones forestales en áreas con aptitud forestal son las principales iniciativas que deben ser realizadas.

Entre las especies recomendadas para la reforestación se encuentra la Bolaina blanca (Guazuma Crinita Martius). Se caracteriza por ser típica de bosques secundarios y por poseer rápido crecimiento, posee fuste cilíndrico-recto sin bifurcaciones y puede llegar a tener entre $25 \mathrm{~cm}-28 \mathrm{~cm}$ y de $15 \mathrm{a} 30 \mathrm{~m}$ de diámetro y de altura, respectivamente (REYNEL et al., 2003). Debido a sus características de trabajabilidad, la madera es usada en la la construcción de casas prefabricadas, en cajonería rústica y fina (embalajes para frutas), en carpintería de puertas y ventanas, fabricación de mondadientes, palitos para chupetes y fósforos, y juguetería (IIAP, 2007).

Para el establecimiento y éxito de las plantaciones forestales diversos factores tienen que ser tomados en cuenta, dentro ellos está la elección de la especie, la calidad de la semilla, el clima, tipo de suelo, tratamientos silviculturales, entre otros. En relación a la semilla, Dias et al. (2012) citan que la propagación por semilla sexual resulta en plantas desuniformes y sujetas a baja calidad en virtud de la gran variación genotípica, lo que puede ser perjudicial para la productividad de las plantaciones. En el caso de G. crinita Paredes et al. (2010), indican que la escasez de semilla de calidad genética apropiada es una de las desventajas para masificar eficientemente las plantaciones forestales con esta especie.

Frente a esta situación, Bandeira et al. (2007) y Xavier et al. (2009), citan que el uso de la propagación vegetativa para la producción de plantas proporciona beneficios al sector forestal porque evita la variabilidad genética, forma plantaciones clonales más productivas y con mejor calidad de la madera. Entre las técnicas de propagación asexual, la miniestaca es una alternativa para la propagación de especies, pudiendo ser utilizadas para fines comerciales, así como auxiliar en el rescate y conservación de los recursos genéticos forestales (DIAS et al., 2012).

No obstante, para el éxito de este método diversos factores como sustrato, invernadero, tipo de propágulo, tipo y dosis de hormona de enraizamiento, recipientes, especie a propagar, etc.; tienen que ser considerados (GUERRA et al., 2018). En lo referente a los invernaderos tienen que proveer alta humedad relativa entre de 80 y $90 \%$ y temperatura adecuada entre 30 y $35^{\circ} \mathrm{C}$ sin oscilaciones bruscas (XAVIER et al., 2009). En relación al sustrato puede ser variado, desde que tenga una buena porosidad y capacidad de retención de agua, y buena agregación al sistema radicular a ser formado (TIMM et al., 2015).

En ese contexto, con la finalidad de generar tecnologías apropiadas de propagación vegetativa y disponer de semilla asexual de calidad en el corto plazo, este trabajo tuvo por objetivo determinar el mejor invernadero, sustrato y aditivo para el enraizamiento de miniestacas de G. crinita M.

\section{MATERIAL Y MÉTODOS}

\section{Localización}

EL estudio fue realizado entre los meses de enero y diciembre de 2016 en las instalaciones de la empresa ASSESSFOR SAC, ubicada en el centro poblado de San Alejandro, distrito de Irazola, provincia de Padre Abad, Región de Ucayali, Perú; localizado en las coordenadas UTM: 8830966 Norte y 75222144 Este, a 217 m.s.n.m. El clima de la región, según la clasificación climática de Köppen-Geiger es tropical, con una precipitación pluvial de $4471 \mathrm{~mm}$, humedad relativa de 82\%, temperatura media de $24.98^{\circ} \mathrm{C}$ y velocidad del viento de $1.4 \mathrm{~m} / \mathrm{s}$ (SENAMHI, 2016). 
Torres et al. - Enraizamiento de miniestacas de Guazuma crinita M. utilizando

diferentes invernaderos, sustratos y aditivos

\section{Obtención de estaquillas de Guazuma crinita M.}

En el vivero se disponía de 20000 plantas de G. crinita, procedentes de semilla botánica seleccionada y certificada de huertos semilleros. Estas, fueron producidas con sustrato a base de estiércol de gallina+cascarilla de arroz carbonizada en proporción 1:1, con riego por microaspersión a cada 8 horas por un periodo de 20 minutos y en condiciones de sombra (50\%) con malla raschel ${ }^{\circledR}$. Al momento de instalar el ensayo las plantas poseían dos meses de edad y $45 \mathrm{~cm}$ de altura. Para el experimento fueron seleccionadas 500 plantas en base a su calidad morfológica (sanas, vigorosas, libre de plagas, y sin síntomas de deficiencia nutricional). Estas fueron podadas a $25 \mathrm{~cm}$ de altura y después de 20 días fueron colectados los brotes.

Las miniestacas fueron obtenidas de acuerdo a la metodología propuesta por Mesen (1998), de este modo, fueron obtenidas de la parte media, eliminándose la parte apical y basal del brote por ser muy suculenta y lignificada, respectivamente. Las miniestacas fueron de $7 \mathrm{~cm}$ de longitud, $4 \mathrm{~mm}$ de diámetro y con 2/3 de área foliar $\left(20-30 \mathrm{~cm}^{2}\right)$. Adicionalmente, fueron previamente desinfectadas por 15 minutos en solución fungicida CUPRAVIT ${ }^{\circledR}(0.3 \%)$. La obtención e instalación de las miniestacas en los ambientes de enraizamiento fue de 1 hora con 10 minutos, utilizando mano de obra capacitada de cuatro personas y la instalación del experimento fue realizada en las primeras horas de la mañana ( 6 a 8 am)

\section{Diseño experimental}

Los tratamientos fueron dispuestos en Diseño de Bloques Completamente al Azar (DBCA) en esquema de parcelas subsubdivididas, con tres bloques y 10 miniestacas por unidad experimental. Las parcelas estuvieron constituidas por dos tipos de invernaderos Invernadero 1: [malla raschel ${ }^{\circledR}$ (50\%)] e invernadero 2: [policarbonato]; las sub parcelas estuvieron constituidas por tres tipos de sustratos: S1) Arena fina, S2) Sistema aeropónico (tecnoport), S3) jiffy pellet ${ }^{\circledR}$ (sustrato compuesto de fibra de coco con musgo comprimidos en una malla fina, suave y biodegradable); y las sub sub parcelas estuvieron compuestas por 4 tipos de aditivos enraizantes: A1) control (sin aditivo), A2) Cicatrizante hormonal sanix ${ }^{\circledR}$, A3) Enraizante AIB a 3000 ppm (Rapid $\operatorname{root}^{\circledR}$ ) y A4) $\operatorname{rapid} \operatorname{root}^{\circledR}+$ sanix $^{\circledR}$

Las miniestacas fueron colocadas a una densidad de $5 \times 5 \mathrm{~cm}$ y a $2 \mathrm{~cm}$ en cada uno de los tratamientos. Es necesario mencionar que, antes de usar el sustrato arena, fue esterilizada a vapor para eliminar cualquier agente contaminante. El producto rapid $\operatorname{root}^{\circledR}$, conteniendo la hormona de enraizamiento AIB fue colocado en la base de las miniestacas en forma de polvo. El sustrato jiffy ${ }^{\circledR}$ antes de ser utilizado fue previamente hidratado por un periodo de 8 horas.

\section{Características de los invernaderos}

Fueron utilizados dos invernaderos, el primero fue construido utilizando en la base estructuras de metal, cubierto con láminas de policarbonato transparente y el segundo también con estructura de metal y con cubierta de malla raschel ${ }^{\circledR}$ color negro con $50 \%$ de sombreamiento (doble capa).

Para tener mayor control de la variación de la temperatura externa, en cada invernadero fueron colocadas bancadas y sobre ellas una estructura pequeña construida con arcos de metal y forrada totalmente con plástico trasparente (mini túnel) con la finalidad de crear una cámara húmeda y condiciones adecuadas de temperatura. Así mismo, de acuerdo a las recomendaciones de Hartmann et al. (2011) en cada mini túnel fue colocado un sistema de riego automático de aspersión nebulizada. En seguida, en estas estructuras fueron acondicionados las miniestacas de G. crinita según la distribución de los tratamientos

\section{Variables evaluadas.}

Después de 20 días, fue evaluado por única vez el porcentaje de enraizamiento (\%), sobrevivencia $(\%)$ y brotes (\%); así como el número $\left(\mathrm{N}^{\circ}\right)$ y longitud $(\mathrm{cm})$ de brotes y raíces en las miniestacas de G. crinita con regla milimetrada. Así, también fueron registrados de los datos de temperatura media, mínima y máxima $\left({ }^{\circ} \mathrm{C}\right)$, humedad relativa (\%) y luminosidad (lux) con termohigrómetro y luxómetro, respectivamente.

\section{Análisis de datos}

Los datos fueron sometidos a la prueba de normalidad Kolmogorov-Smirnov. Después de verificado la distribución normal, los datos fueron sometidos a análisis de varianza (ANOVA) y las medias de los tratamientos fueron comparados con la prueba de Scott-Knott $(\mathrm{P} \leq 0,05)$ utilizando el programa Infostat (DI RIENZO et al., 2008). 


\section{RESULTADOS Y DISCUSIÓN}

Durante la conducción del ensayo fueron registrados en media, mínima y máxima: 31, 8; 28,3 y 33, $8^{\circ} \mathrm{C}$ de temperatura; 84, $8 ; 70,3$ y 93,3\% de humedad relativa y 9834; 4027 y 13622 lux de intensidad lumínica, respectivamente, dentro del mini túnel ubicado en el invernadero construido con cubierta de policarbonato. De la misma forma, en el invernadero con cubierta de malla raschel ${ }^{\circledR}$ fue registrado en media, mínima y máxima: 30,$8 ; 26,9$ y $33,8{ }^{\circ} \mathrm{C} ; 77,5 ; 59,4$ y $93,7 \%$ de humedad relativa y 7339 ; 3035 y 16163 lux, respectivamente.

El Análisis de varianza mostró interacción significativa entre los invernaderos, sustratos y aditivos según la prueba de F ( $\mathrm{p} \leq 0,05)$ para las variables porcentaje de enraizamiento, porcentaje de sobrevivencia, número de raíces y número de brotes en las miniestacas de G. crinita. De otro lado, solamente hubo interacción significativa para las variables longitud de raíces y de brotes por efecto de tipos invernaderos y sustratos.

\section{Enraizamiento (\%)}

El porcentaje de enraizamiento de las miniestacas de G. crinita, fue favorecido en los dos invernaderos. No entanto, resultados superiores de 93,3 hasta 100\% fueron obtenidos en el invernadero de policarbonato asociado con el sustrato arena fina (S1) con y sin aditivo enraizante. De otra parte, en el invernadero de malla raschel ${ }^{\circledR}$, fue constatado que el sustrato arena fina (S1) combinado con el aditivo sanix $^{\circledR}$ (A2) y el sustrato jiffy ${ }^{\circledR}$ (S3) asociado al aditivo rapid $\operatorname{root}^{\circledR}$ (AIB en dosis de 3000 ppm en polvo) (A3) presentaron los mejores resultados de 96,7 y 100\% de enraizamiento, respectivamente. De manera opuesta, se observa que los menores resultados fueron verificados cuando fue utilizado el sistema aeropónico (S2) con y sin aditivo enraizante en los dos invernaderos, obteniendo valores entre 16 y $70 \%$ de enraizamiento (Tabla 1 ).

Tabla 1. Porcentaje de enraizamiento de miniestacas de G. crinita M. por efecto de la interacción entre invernaderos (malla y policarbonato), sustratos (S1 - Arena fina; S2 - sistema aeropónico; S3 - jiffy ${ }^{\circledR}$ ) y aditivos (A1 - sin aditivo/control; A2 - sanix ${ }^{\circledast}$; A3 - rapid $\operatorname{root}^{\circledast} ;$ A4 - rapid root + sanix ${ }^{\circledR}$ ) en San Alejandro, Irazola, Padre Abad, Ucayali, Perú.

Table 1. Rooting percentage of minicuttings of G. crinita M. by effect to interaction between greenhouses (mesh and polycarbonate), substrates (S1 - fine sand, S2 - aeroponic system, S3 - jiffy ${ }^{\circledR}$ ) and additives (A1 - without additive/control; A2 - sanix ${ }^{\circledast}, A 3$ - rapid $\operatorname{root}^{\circledR}, A 4$ - rapid root + sanix $\left.{ }^{\circledR}\right)$ in San Alejandro, Irazola, Padre Abad, Ucayali, Peru.

\begin{tabular}{|c|c|c|c|c|c|c|c|c|c|c|}
\hline \multirow{2}{*}{ Invernaderos } & & \multicolumn{8}{|c|}{ Enraizamiento (\%) } & \multirow{2}{*}{ Media } \\
\hline & & A1 & & A2 & & A3 & & A4 & & \\
\hline \multirow{3}{*}{ Malla raschel ${ }^{\circledR}$} & S1 & 83,3 & Bya & 96,7 & Axa & 80,0 & Byb & 83,3 & Bya & 85,8 \\
\hline & $\mathrm{S} 2$ & 16,7 & Cyb & 16,7 & Сус & 70,3 & $A x c$ & 30,0 & Byb & 33,4 \\
\hline & S3 & 86,7 & Bxa & 86,7 & $\mathrm{Bxb}$ & 100 & Axa & 80,0 & Cya & 88,3 \\
\hline \multirow{5}{*}{ Policarbonato } & Media & 62,2 & & 66,7 & & 83,4 & & 64,4 & & \\
\hline & S1 & 96,7 & Axa & 93,3 & Axa & 100 & Axa & 96,7 & Axa & 96,7 \\
\hline & $\mathrm{S} 2$ & 30,0 & Cxc & 63,3 & $A x c$ & 50,0 & Byc & 60,0 & $A x c$ & 50,8 \\
\hline & S3 & 73,3 & Cyb & 80,0 & Byb & 80,0 & Byb & 86,7 & $A x b$ & 80,0 \\
\hline & Media & 66,7 & & 78,9 & & 76,7 & & 81,1 & & \\
\hline
\end{tabular}

* En cada invernadero, medias seguidas de la misma letra mayúscula en la línea y minúscula en la columna ("A" "a”) no presentan diferencias estadísticas significativas por la prueba de Scott-Knott a $5 \%$ de probabilidad. Del mismo modo, medias iguales ' $x$ ' e ' $y$ ' no difieren entre invernaderos. Los CVs fueron: CVs (\%) foram: CVI = I4,3\%; CV2=I2,2\%; CV $3=11,6 \%$

De acuerdo con Leakey et al. (1990), un enraizamiento por debajo del 70\% se considera perjudicial a nivel comercial para cualquier especie. En ese sentido, en este trabajo, el sistema aeropónico no influenció positivamente en el enraizamiento de miniestacas de G. crinita, probablemente porque estuvieron en un medio sin sustrato. Al respecto, Timm et al. (2015), mencionan que, para formar el sistema radicular en las estacas es necesario un sustrato que presente buena porosidad, capacidad de retener de agua y buena agregación. No entanto, Ritter et al. (2001), indican que el sistema aeropónico mostró ser eficiente para la producción de semillas de papa, porque según, Nichols (2005) es una tecnología que no usa suelo, puesto que el sistema radicular se desarrolla en un invernadero oscuro, que continuamente es saturado con micro gotas de solución nutritiva.

Resultados similares a los determinados en este estudio, fueron reportados por Angulo (2016), trabajando en la propagación vegetativa de Plukenetia polyadenia utilizando arena fina combinado 
con AIB en cámaras de subirrigación. También, Huamán (2015) determinó 98\% de enraizamiento en estacas de Coffea arabica L. en sustrato jiffy ${ }^{\circledR}$. Doina et al. (2015) y Valverde-Cerdas et al. (2004), utilizando sustrato jiffy ${ }^{\circledR}$ lograron 100 y $90 \%$ de enraizamiento en brotes de Gmelina arborea y Blackberry.

En relación a la hormona AIB, Vernier y Cardoso (2013), indican que esta sustancia promueve y favorece el enraizamiento de estacas y las dosis van a depender de la especie y de los tipos y tamaños de estacas. De esta manera, Lana et al. (2008) determinaron que las dosis entre $2000 \mathrm{mg} \mathrm{L}^{-1}$ y $5000 \mathrm{mg} \mathrm{L}^{-1}$ proporcionaron respuestas significativas sobre el sistema radicular en estacas de Eucalyptus urophylla.

En este estudio posiblemente la aplicación de la hormona en polvo también favoreció el enraizamiento, porque de acuerdo a Cuquel y Minami (1994) y Fachinello et al. (2005) señalan que la aplicación vía polvo presenta facilidad en la preparación, es más durable y más eficiente porque posee mayor capacidad de adherencia e impregnación en la base de la estaca.

Como fue observado el sustrato arena fina dio resultados satisfactorios. Al respecto, Mesén (1998), asegura que es un excelente sustrato para el enraizamiento de la mayoría de las especies forestales porque posee excelentes características de aireación, anclaje, durabilidad y además el agua retenida por las partículas de arena es fácilmente absorbida por las raíces. En el mismo sentido, Soudre et al. (2011) señalan que la arena es un sustrato económico, disponible localmente y fácil de esterilizar.

Así mismo, las características del sustrato jiffy ${ }^{\circledR}$, también influenciaron de manera eficiente en los resultados, pues Hernández et al. (2006) citan que este sustrato se caracteriza por poseer condiciones ideales de $\mathrm{pH}(5,3)$, pequeñas trazas de cal dolomita y elementos minerales como: calcio $(21 \%$ de Ca y $30 \%$ de $\mathrm{CaO}$ ), Magnesio (12,5\% de $\mathrm{Mg}$ y $21 \%$ de $\mathrm{MgO}$ ) y pequeñas cantidades de microelementos como Fe y $\mathrm{Cu}$.

No obstante, otro factor que influyó positivamente en en el enraizamiento de las miniestacas en el invernadero de policarbonato fueron las adecuadas condiciones de temperatura y humedad relativa, pues, Murillo-Gamboa et al. (2013) y Gatti et al. (2011) señalan que estos factores deben estar entre 30 a $35^{\circ} \mathrm{C}$ y de 80 a $90 \%$, respectivamente. Por el contrario, en el invernadero con cubierta de malla raschel ${ }^{\circledR}$ hubo mayor variación de la temperatura y humedad relativa llegando hasta un máximo y mínimo de de $26{ }^{\circ} \mathrm{C}$ y $59,4 \%$, respectivamente, lo que pudo ocasionar mayor tasa de evapotranspiración por existir menor concentración de vapor de agua en el ambiente, causando deshidratación y muerte de las miniestacas.

Otro factor que tiene ser considerado es la adecuada intensidad de luz dentro del invernadero, en ese sentido, nótese que en el invernadero con cobertura de policarbonato fue registrado mayor media y menor valor de 9834 y 13622 lux, frente a 7339 y 16163 lux en el invernadero malla raschel ${ }^{\circledR}$, respectivamente. Al respecto, Carvalho (1996) cita que las plantas utilizan eficientemente la luz, cuando sus hojas son iluminadas uniformemente con intensidades bajas de luz, en relación a las que quedan supersaturadas por intensidades altas, o permanecen en profunda sombra.

\section{Sobrevivencia de miniestacas (\%)}

Los mejores resultados fueron obtenidos en los dos invernaderos de malla raschel ${ }^{\circledR} \mathrm{y}$ de policarbonato asociados con los sustratos arena fina y jiffy ${ }^{\circledR}$ con y sin aditivos enraizantes, alcanzado hasta $100 \%$ de miniestacas vivas (Tabla 2).

Resultados similares fueron obtenidos por Yamamoto et al. (2010) trabajando con el enraizamiento de estacas de Psidium guajava L. utilizando AIB en polvo. No obstante, nótese que, en el invernadero de malla raschel ${ }^{\circledR}$ asociado con el medio de enraizamiento aeropónico y con el aditivo sanix ${ }^{\circledR}$ presentó los menores porcentajes de sobrevivencia. De este modo, queda demostrado que, el uso de un medio para enraizamiento sin sustrato es perjudicial para la sobrevivencia de miniestacas de G. crinita, además las altas temperaturas y baja humedad relativa de $26{ }^{\circ} \mathrm{C}$ y $59,4 \%$, registrada en el ambiente también contribuyó con estos resultados.

\section{Miniestacas con brotes (\%)}

Los menores resultados fueron obtenidos en el invernadero malla raschel ${ }^{\circledR}$ asociado con el sistema aeroponico sin aditivo y con los aditivos sanix ${ }^{\circledR}$ y rapid root $^{\circledR}+$ sanix $^{\circledR}$ con valores de 83,3; 50 y 86,7\%, respectivamente. No entanto con el uso del aditivo rapid root en este ambiente se obtuvo $100 \%$ de brotación. En el caso del aditivo sanix ${ }^{\circledR}$ era de esperarse, pues solamente el $50 \%$ de las miniestacas sobrevivieron. De otro lado, se observa que los sustratos arena fina y jiffy ${ }^{\circledR}$ combinados con y sin aditivo en los dos invernaderos de malla raschel ${ }^{\circledR}$ y policarbonato provocaron $100 \%$ de brotamiento en las miniestacas de G. crinita (Tabla 3). 
Tabla 2. Porcentaje de sobrevivencia de miniestacas de G. crinita M. por efecto de la interacción entre invernaderos (malla raschel ${ }^{\circledR}$ y policarbonato), substratos (S1 - arena fina; S2 - sistema aeropónico; S3 - jiffy ${ }^{\circledR}$ ) y aditivos (A1 - sin aditivo/control; A2 - sanix ${ }^{\circledR} ; A 3-\operatorname{rapid} \operatorname{root}^{\circledR} ; A 4-\operatorname{rapid} \operatorname{root}^{\circledR}+\operatorname{sanix}^{\circledR}$ ) en San Alejandro, Irazola, Padre Abad, Ucayali, Perú.

Table 2. Percentage of survival of minicuttings of G. crinita M. by effect to the interaction between greenhouses (raschel ${ }^{\circledR}$ and polycarbonate mesh), substrates (S1 - fine sand, S2 - aeroponic system, S3 - jiffy ${ }^{\circledR}$ ) and additives (A1 - without additive / control; A2 - sanix ${ }^{\circledR}, A 3-\operatorname{rapid} \operatorname{root}^{\circledR}, A 4-\operatorname{rapid} \operatorname{root}^{\circledR}+\operatorname{sanix}^{\circledR}$ ) in San Alejandro, Irazola, Padre Abad, Ucayali, Peru..

\begin{tabular}{|c|c|c|c|c|c|c|c|c|c|c|}
\hline \multirow{2}{*}{ Invernaderos } & & \multicolumn{8}{|c|}{ Sobrevivencia de estacas (\%) } & \multirow{2}{*}{ Media } \\
\hline & & A1 & & A2 & & A3 & & A4 & & \\
\hline \multirow{3}{*}{ Malla raschel ${ }^{\circledR}$} & S1 & 100 & Axa & 100 & Axa & 100 & Axa & 100 & Axa & 100 \\
\hline & S2 & 83,3 & Byb & 50,0 & Cyb & 96,7 & Axa & 83,3 & Byb & 78,3 \\
\hline & S3 & 100 & Axa & 100 & Axa & 100 & Axa & 100 & Axa & 100 \\
\hline \multirow{5}{*}{ Policarbonato } & Media & 94,4 & & 83,3 & & 98,9 & & 94.4 & & \\
\hline & S1 & 100 & Axa & 100 & Axa & 100 & Axa & 100 & Axa & 100 \\
\hline & S2 & 93,3 & $\mathrm{Bxb}$ & 100 & Axa & 100 & Axa & 100 & Axa & 98,3 \\
\hline & S3 & 100 & Axa & 96,7 & Axa & 96,7 & Axa & 100 & Axa & 98,3 \\
\hline & Media & 97,8 & & 98,9 & & 98,9 & & 100 & & \\
\hline
\end{tabular}

* En cada invernadero, medias seguidas de la misma letra mayúscula en la línea y minúscula en la columna ("A" "a") no presentan diferencias estadísticas significativas por la prueba de Scott-Knott a $5 \%$ de probabilidad. Del mismo modo, medias iguales ' $x$ ' e ' $y$ ' no difieren entre invernaderos. Los CVs fueron: CVI =29,87\%; CV2=12,2\%; CV3 $=11,6 \%$

Tabla 3. Porcentaje de brotes en miniestacas de G. crinita M. por efecto de la interacción entre invernaderos (malla y policarbonato), substratos (S1 - arena fina; S2 - sistema aeropónico; S3 - jiffy ${ }^{\circledR}$ ) y aditivos (A1 - sin aditivo/control; A2 - sanix $\left.{ }^{\circledR} ; A 3-\operatorname{rapid} \operatorname{root}^{\circledast} ; A 4-\operatorname{rapid} \operatorname{root}^{\circledR}+\operatorname{sanix}^{\circledR}\right)$ en San Alejandro, Irazola, Padre Abad, Ucayali, Perú.

Table 3. Percentage of shoots in minicuttings of G. crinita M. by effect to the interaction between greenhouses (mesh and polycarbonate), substrates (S1 - fine sand, S2 - aeroponic system, S3 - jiffy ${ }^{\circledR}$ ) and additives (A1 - without additive/control; A2 - sanix $x^{\circledR} ; A 3$ - rapid $\left.\operatorname{root}^{\circledR} ; A 4-\operatorname{rapid} \operatorname{root}^{\circledast}+\operatorname{sanix}^{\circledast}\right)$ in San Alejandro, Irazola, Padre Abad, Ucayali, Peru

\begin{tabular}{|c|c|c|c|c|c|c|c|c|c|c|}
\hline \multirow{2}{*}{ Invernaderos } & & \multicolumn{8}{|c|}{ Porcentaje de estacas con brotes } & \multirow{2}{*}{ Media } \\
\hline & & A1 & & A2 & & A3 & & A4 & & \\
\hline & S1 & 100 & Axa & 100 & Axa & 100 & Axa & 100 & Axa & 100 \\
\hline \multirow[t]{4}{*}{ Malla raschel ${ }^{\circledR}$} & S2 & 83,3 & Byb & 50,0 & Cyb & 100 & Axa & 86,7 & Byb & 80,0 \\
\hline & S3 & 100 & Axa & 100 & Axa & 100 & Axa & 100 & Axa & 100 \\
\hline & Media & 94,4 & & 83,3 & & 100 & & 95,6 & & \\
\hline & S1 & 100 & Axa & 100 & Axa & 100 & Axa & 100 & Axa & 100 \\
\hline \multirow[t]{3}{*}{ Policarbonato } & S2 & 93,3 & $B \times b$ & 100 & Axa & 100 & Axa & 100 & Axa & 98,3 \\
\hline & S3 & 100 & Axa & 100 & Axa & 100 & Axa & 100 & Axa & 100 \\
\hline & Media & 97,8 & & 100 & & 100 & & 100 & & \\
\hline
\end{tabular}

* En cada invernadero, medias seguidas de la misma letra mayúscula en la línea y minúscula en la columna ("A" "a") no presentan diferencias estadísticas significativas por la prueba de Scott-Knott a $5 \%$ de probabilidad. Del mismo modo, medias iguales ' $x$ ' e ' $y$ ' no difieren entre invernaderos. Los CVs fueron: CVI =24,45\%; CV2=24,45\%; CV3=9,5\%

Nótese que, el porcentaje de enraizamiento fue directamente proporcional con el porcentaje de brotación, es decir que donde hubo mayor porcentaje de enraizamiento hubo mayor porcentaje de miniestacas brotadas y viceversa. Mesén (1998), indica que, la formación de raíces, recupera el balance hídrico y consecuentemente las reacciones fotosintéticas, puesto que existe un balance de crecimiento entre el brote y las raíces. En el mismo sentido, Hartmann et al. (2011) y Tchoundjeu et al. (2002) citan que, la emisión de brotes en las estacas durante el proceso de enraizamiento es esencial porque es en estos órganos donde se produce auxinas naturales, además de carbohidratos, que son transportados hacia la parte inferior de las estacas para estimular el enraizamiento.

\section{Número y longitud de brotes}

En la Tabla 4 se observa que el mayor número de brotes fue obtenido en arena fina y jiffy ${ }^{\circledR}$ asociados al aditivo rapid $\operatorname{root}^{\circledR} \operatorname{con} 4,3$ y 3,9 brotes en promedio, respectivamente. No obstante, los menores resultados fueron verificados en el invernadero de malla raschel ${ }^{\circledR}$ asociado al sistema aeroponico con los aditivos sanix ${ }^{\circledR}$ y rapid root ${ }^{\circledR}$ más sanix $^{\circledR}$ con 1,3 y 2,1 brotes, respectivamente. 
Torres et al. - Enraizamiento de miniestacas de Guazuma crinita M. utilizando

diferentes invernaderos, sustratos y aditivos

Tabla 4. Número de brotes en miniestacas de G. crinita M. por efecto de la interacción entre invernaderos (malla y policarbonato), substratos (S1 - arena fina; S2 - sistema aeropónico; S3 - jiffy ${ }^{\circledR}$ ) y aditivos (A1 - sin aditivo/control; A2 - sanix ${ }^{\circledR}$ A3 - rapid $\operatorname{root}^{\circledR} ; A 4-\operatorname{rapid} \operatorname{root}^{\circledR}+\operatorname{sanix}^{\circledR}$ en en San Alejandro, Irazola, Padre Abad, Ucayali, Perú.

Table 4. Number of shoots in minicuttings of G. crinita M. by effect to the interaction between greenhouses (mesh and polycarbonate), substrates (S1-fine sand, S2-aeroponic system, S3-jiffy ${ }^{\circledR}$ ) and additives (A1-without additive/control; A2 - sanix ${ }^{\circledR} ; A 3$ - rapid $\operatorname{root}^{\circledR} ;$ A4 - rapid $\operatorname{root}^{\circledR}+$ sanix $^{\circledR}$ in San Alejandro, Irazola, Padre Abad, Ucayali, Peru.

\begin{tabular}{|c|c|c|c|c|c|c|c|c|c|c|}
\hline \multirow{2}{*}{ Invernaderos } & & \multirow[b]{2}{*}{ A1 } & & \multicolumn{5}{|c|}{ Número de brotes } & & \multirow{2}{*}{ Media } \\
\hline & & & & $\mathrm{A} 2$ & & A3 & & A4 & & \\
\hline \multirow{3}{*}{ Malla raschel ${ }^{\circledR}$} & S1 & 3,1 & Aya & 3,2 & Axa & 3,1 & Aya & 3,0 & Aya & 3,1 \\
\hline & $\mathrm{S} 2$ & 2,3 & $\mathrm{Bxb}$ & 1,3 & Cyb & 3,0 & Axa & 2,1 & Byb & 2,2 \\
\hline & S3 & 3,2 & Aya & 3,1 & Axa & 3,1 & Aya & 3,1 & Aya & 3,1 \\
\hline \multirow{5}{*}{ Policarbonato } & Media & 2,9 & & 2,5 & & 3,1 & & 2,7 & & \\
\hline & S1 & 3,6 & $\mathrm{Bxb}$ & 3,5 & Bxa & 4,3 & Axa & 3,8 & Bxa & 3,8 \\
\hline & $\mathrm{S} 2$ & 2,8 & $\mathrm{Bxc}$ & 3,1 & Bxa & 3,4 & $A x b$ & 3,6 & Axa & 3,2 \\
\hline & S3 & 4,3 & Axa & 3,4 & Bxa & 3,9 & Axa & 3,8 & Bxa & 3,8 \\
\hline & Media & 3,6 & & 3,3 & & 3,9 & & 3,7 & & \\
\hline
\end{tabular}

* En cada invernadero, medias seguidas de la misma letra mayúscula en la línea y minúscula en la columna ("A" "a") no presentan diferencias estadísticas significativas por la prueba de Scott-Knott a $5 \%$ de probabilidad. Del mismo modo, medias iguales ' $x$ ' e ' $y$ ' no difieren entre invernaderos. Los CVs fueron: CVI =37,38\%; CV2=54,8I\%; CV3=27,96\%

En relación a la longitud $(\mathrm{cm})$ de los brotes, fue determinado que solamente hubo efectos significativos para la interacción entre invernaderos y sustratos. Además, nótese que el sistema aeropónico y sustrato jiffy ${ }^{\circledR}$ en ambos invernaderos no presentaron diferencias estadísticas significativas $(\mathrm{p} \leq 0,05)$ sobre esta variable. No obstante, fueron verificados miniestacas con brotes de $28,8 \mathrm{~cm}$ de longitud

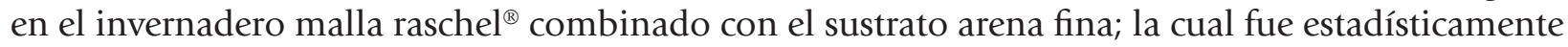
superior a la la longitud de brotes obtenidos en el invernadero de policarbonato que alcanzó $19.9 \mathrm{~cm}$ en promedio (Tabla 5).

Tabla 5. Longitud de brotes en miniestacas de Guazuma crinita M. por efecto de la interacción entre los invernaderos (malla y policarbonato) y los substratos (S1 - arena fina; S2 - sistema aeropónico; S3 - jiffy ${ }^{\circledR}$ y aditivos

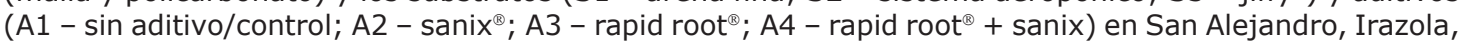
Padre Abad, Ucayali, Perú.

Table 5. Length of shoots in minicuttings of G. crinita M. by effect to the interaction between greenhouses (mesh and polycarbonate) and substrates (S1-fine sand, S2-aeroponic system, S3 - jiffy ${ }^{\circledR}$ ) and additives (A1 - without

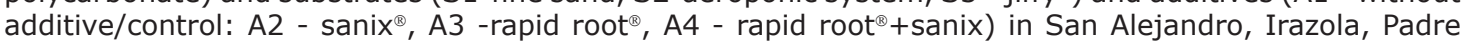
Abad, Ucayali, Peru.

\begin{tabular}{lccc}
\hline & \multicolumn{2}{c}{ Invernaderos Long. de brotes $(\mathbf{c m})$} & \\
\hline \multirow{3}{*}{ Malla raschel $^{\circledR}$} & $\mathrm{S} 1$ & 28,8 & $\mathrm{Xa}$ \\
& $\mathrm{S} 2$ & 11,5 & $\mathrm{Xb}$ \\
& $\mathrm{S} 3$ & 17,1 & $\mathrm{Xb}$ \\
Policarbonato & Media & 19,1 & $\mathrm{Ya}$ \\
& $\mathrm{S} 1$ & 19,9 & $\mathrm{Xa}$ \\
& $\mathrm{S} 2$ & 16,2 & $\mathrm{Xa}$
\end{tabular}

*Medias seguidas de la misma letra minúscula en la columna (“a”) no presentan diferencias estadísticas significativas por la prueba de Scott-Knott a $5 \%$ de probabilidad en los sustratos. Del mismo modo, medias iguales ' $x$ ' e ' $y$ ' no difieren entre invernaderos. Los CVs fueron: CvI = $21,5 \%$; $\mathrm{Cv} 2=15,6$ e $\mathrm{Cv} 3=18,3 \%$.

Sin duda la presencia de hojas en las miniestacas de G.crinita promovió la formación de brotes. Resultados opuestos fueron determinados por Pio et al. (2004) trabajando con estacas de estacas de Figuera (Ficus carica L.), dado que las estacas sin hojas presentaron baja taza de brotación. Otro de los factores que contribuyó a la formación de brotes fue el uso de la sección media del brote, pues Chagas et al. (2008) explican que la remoción del ápice caulinar, normalmente resulta en el crecimiento de yemas laterales, explicando la mayor brotación en las estacas de las secciones medias y basales debido a la ausencia de la actividad de las auxinas removidas en el ápice. Lo contrario ocurre en las estacas apicales, pues estas concentran alta cantidad de auxina endógena regulando la dominancia apical e inhibiendo el crecimiento de yemas axilares (SANTOS et al., 2016) 
Por otro lado, Hartmann, Kester y Davies, (1990), citan que es importante el desarrollo de la parte aérea porque es uno de los factores fundamentales para la emisión de raíces en las estacas, visto que la producción de fitohormonas como el ácido indolacético y de cofactores esenciales para el proceso de enraizamiento se da a través de las hojas.

\section{Número y longitud de raíces}

De manera general en la Tabla 6 se observa, que el sistema aeropónico asociado con y sin aditivos enraizantes en los dos invernaderos provocó menos de 4,6 de raíces en las miniestacas de G. crinita, resultando estadísticamente inferior $(\mathrm{p} \leq 0,05)$ a los otros tratamientos.

Tabla 6. Número de raíces en miniestacas de G. crinita M. por efecto de los invernaderos (malla y policarbonato), substratos (S1 - arena fina; S2 - sistema aeropónico; S3 - jiffy ${ }^{\circledR}$ ) y aditivos (A1 - sin aditivo/control; A2 - sanix ${ }^{\circledR} ;$ A3 - rapid $\operatorname{root}^{\circledR} ;$ A4 - rapid $\left.\operatorname{root}^{\circledR}+\operatorname{sani}^{\circledR}\right)$ en San Alejandro, Irazola, Padre Abad, Ucayali, Perú.

Table 6. Number of roots in minicuttings of G. crinita M. by effect to greenhouse effect (mesh and polycarbonate), substrates (S1 - fine sand, S2 - aeroponic system, S3 - jiffy ${ }^{\circledR}$ ) and additives (A1 - without additive / control;

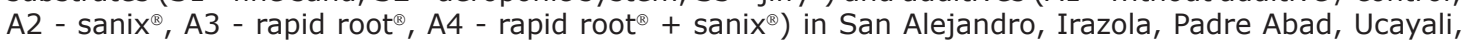
Peru.

\begin{tabular}{|c|c|c|c|c|c|c|c|c|c|c|}
\hline \multirow{2}{*}{ Invernadero } & & \multicolumn{8}{|c|}{ Número de raíces } & \multirow{2}{*}{ Media } \\
\hline & & A1 & & A2 & & A3 & & A4 & & \\
\hline \multirow{3}{*}{ Malla raschel ${ }^{\circledR}$} & $\mathrm{S1}$ & 6,0 & Byb & 10,1 & Axa & 6,0 & Byb & 6,9 & Byb & 7,2 \\
\hline & $\mathrm{S} 2$ & 0,7 & $\mathrm{Bxc}$ & 0,8 & $B \times b$ & 4,6 & $A x b$ & 1,1 & $\mathrm{Bxc}$ & 1,8 \\
\hline & S3 & 12,3 & Axa & 12,0 & Axa & 14,4 & Axa & 14,7 & Axa & 13,3 \\
\hline \multirow{5}{*}{ Policarbonato } & Media & 6,3 & & 7,6 & & 8,3 & & 7,6 & & \\
\hline & $\mathrm{S} 1$ & 11,2 & Bxa & 12,4 & Bxa & 18,9 & Axa & 12,6 & Bxa & 13,8 \\
\hline & $\mathrm{S} 2$ & 1,9 & $A x b$ & 3,9 & Axc & 3,0 & Axc & 3,2 & $A x b$ & 3,0 \\
\hline & S3 & 10,7 & Bxa & 8,8 & $\mathrm{Bxb}$ & 14,5 & $A x b$ & 12,6 & Axa & 11,7 \\
\hline & Media & 7,9 & & 8,4 & & 12,1 & & 9,5 & & \\
\hline
\end{tabular}

* En cada invernadero, medias seguidas de la misma letra mayúscula en la línea y minúscula en la columna ("A" "a”) no presentan diferencias estadísticas significativas por la prueba de Scott-Knott a $5 \%$ de probabilidad. Del mismo modo, medias iguales ' $x$ ' e ' $y$ ' no difieren entre invernaderos. Los CVs fueron: CvI = 22,8\%; Cv2 = I4,2 e Cv3 = $13,3 \%$.

En este caso, probablemente el reducido número de raíces, fue debido a las temperaturas máximas de $33^{\circ} \mathrm{C}$ registradas en los invernaderos, pues según, Otazú (2010) cita que el sistema aeropónico no funciona en invernaderos calientes, puesto que las temperaturas elevadas estimulan altas tasas de transpiración lo cual ocasiona generalmente la deshidratación y muerte de las estacas (FACHINELLO et al., 2005).

Por el contrario, los mejores resultados fueron obtenidos en el invernadero de policarbonato, destacando la combinación: sustrato arena fina con el aditivo enraizante rapid root $^{\circledR}$, con 18,9 raíces, lo cual resultó estadísticamente superior a la misma combinación en el invernadero de malla raschel ${ }^{\circledR}$ que solamente obtuvo 6 raíces.

Estos resultados demuestran que, las mejores condiciones de temperatura y humedad relativa (28,3-33,8 ${ }^{\circ} \mathrm{C}$ y 70,3-93,3\%) en el túnel ubicado en el invernadero de policarbonato, posibilitó la mayor formación de raíces en las miniestacas de G. crinita. Al respecto, Murillo-Gamboa et al. (2013), señalan que las condiciones adecuadas de temperatura y humedad relativa para el enraizamiento son de 30 a $35{ }^{\circ} \mathrm{C}$ y de 80-90\%, respectivamente. Así también, Bortolini et al. (2008), citan que la uniformidad de las raíces, mayor número de raíces y alto índice de enraizamiento en corto período de tiempo, son obtenidas en estacas tratadas principalmente con el fitorregulador AIB.

Otro de los factores que influyó positivamente en la formación de raíces fue la aplicación vía polvo de la hormona AIB contenida en el producto rapid root ${ }^{\circledR}$, pues según Cuquel y Minami (1994) el uso de AIB de forma sólida se adhiere mejor en la base de las estacas y el periodo de exposición es más prolongado en comparación cuando se aplica de forma líquida. Resultados semejantes fueron determinados por Yamamoto et al. (2010), trabajando en el enraizamiento de estacas de Psidium guajava L. Del mismo modo, Leite y Martins (2007), verificaron en media 24,37 raíces en estacas semileñosas de cacao (Theobroma cacao) utilizando $4,5 \mathrm{mg} \mathrm{kg}^{-1}$ de AIB aplicada en forma de polvo.

De otro lado, resultados inferiores, satisfactorios y similares, fueron obtenidos cuando fue

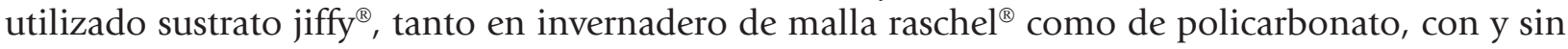




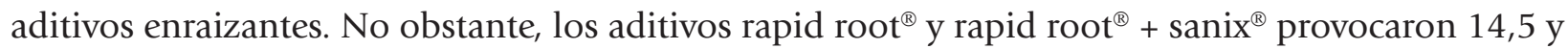
12,6 raíces, respectivamente. Por lo visto, las características de porosidad y mayor retención de agua del sustrato jiffy ${ }^{\circledR}$ combinado con auxinas promotoras de enraizamiento también promovieron la emisión y formación de raíces. Al respecto, Hernández et al. (2006) mencionan que el sustrato jiffy ${ }^{\circledR}$ está elaborado de turba comprimida por lo que es capaz de absorber agua y aire en cantidades adecuadas. Por tal motivo es considerado ideal para la formación de las raíces en estacas de eucalipto (Eucalyptus grandes).

Para la longitud de raíces, fue verificado que no hubo efecto significativo de la interacción triple de los factores (invernadero, sustratos y aditivos enraizantes). No obstante, hubo efectos significativos de los invernaderos y sustratos (Tabla 7). De este modo, el sistema aeroponico dentro de los invernaderos malla raschel ${ }^{\circledR}$ y policarbonato provocó la menor longitud de raíces de 4,9 y 7,4 cm, respectivamente. Sin embargo, las miniestacas instaladas en el sustrato arena fina dentro del invernadero policarbonato emitieron raíces de 46,1 cm de longitud.

Tabla 7. Longitud de raíces en miniestacas de G. crinita M. por efecto de la interacción entre los invernaderos (malla y policarbonato) y substratos (S1 - arena fina; S2-sistema aeropónico; S3 - jiffy ${ }^{\circledR}$ ) en San Alejandro, Irazola, Padre Abad, Ucayali, Perú.

Table 7. Length of roots in minicuttings of G. crinita M. by effect to the interaction between the greenhouses (mesh and polycarbonate) and substrates (S1 - fine sand, S2- $\quad$ aeroponic system, S3 - jiffy ${ }^{\circledR}$ ) in San Alejandro, Irazola, Padre Abad, Ucayali, Peru.

\begin{tabular}{|c|c|c|c|}
\hline Invernaderos & & de ra & \\
\hline \multirow{3}{*}{ Malla raschel ${ }^{\circledR}$} & S1 & 20,5 & $\mathrm{Yb}$ \\
\hline & S2 & 4,9 & $\mathrm{Xc}_{\mathrm{c}}$ \\
\hline & S3 & 41,4 & $\mathrm{Xa}$ \\
\hline \multirow{5}{*}{ Policarbonato } & Media & 22,3 & \\
\hline & S1 & 46,1 & $\mathrm{Xa}$ \\
\hline & $\mathrm{S} 2$ & 7,4 & $X_{c}$ \\
\hline & S3 & 35,3 & $\mathrm{Xb}$ \\
\hline & Media & 29,6 & \\
\hline
\end{tabular}

*Medias seguidas de la misma letra minúscula en la columna ("a”) no presentan diferencias estadísticas significativas por la prueba de Scott-Knott a $5 \%$ de probabilidad en los sustratos. Del mismo modo, medias iguales ' $x$ ' e ' $y$ ' no difieren entre invernaderos. Los CVs fueron: CvI = I8,5\%; $\mathrm{Cv} 2=17,6$ e Cv3 $=19,3 \%$

De la misma manera, el sustrato jiffy ${ }^{\circledR}$, también promovió el crecimiento de raíces, siendo de 41,4 y $35,3 \mathrm{~cm}$ en los invernaderos malla raschel ${ }^{\circledR}$ y policarbonato, respectivamente (Tabla 7). Resultados similares fueron verificados por Echeverría et al. (2014), al trabajar en el enraizamiento de miniestacas de híbridos F1 de café (Coffea arabica L.).

De otro lado, se observaron efectos simples significativos de los aditivos enraizantes sobre la longitud de las raíces. Así, el aditivo rapid root ${ }^{\circledR}$ generó raíces de $30,3 \mathrm{~cm}$ de longitud, demostrando ser estadísticamente superior a los demás aditivos que solamente provocaron en media, raíces con 24,7 cm de longitud (Tabla 8).

Tabla 8. Longitud de raíces en miniestacas de G. crinita M. por efecto de los aditivos A1 - sin aditivo/control; A2-sanix ${ }^{\circledR}$; A3 - rapid root ${ }^{\circledR}$; A4 - rapid root $^{\circledR}+$ sanix $^{\circledR}$ en San Alejandro, Irazola, Padre Abad, Ucayali, Perú.

Table 8. Length of roots in minicuttings of G. crinita M. by effect of the additives A1 - without additive/control; A2-sanix ${ }^{\circledast}$; A3-rapid root $^{\circledast} ;$ A4 - rapid $\operatorname{root}^{\circledR}+\operatorname{sanix}^{\circledast}$ in San Alejandro, Irazola, Padre Abad, Ucayali, Peru.

\begin{tabular}{|c|c|c|c|c|}
\hline \multicolumn{4}{|c|}{$\frac{\text { Longitud de raíces }(\mathrm{cm})}{\text { Aditivos }}$} & \multirow[t]{2}{*}{ Media } \\
\hline $\mathrm{A} 1$ & A2 & A3 & A4 & \\
\hline 22,2 B & $25,8 \mathrm{~B}$ & $30,3 \mathrm{~A}$ & $26,1 \mathrm{~B}$ & 26,1 \\
\hline
\end{tabular}

* Medias seguidas de la misma letra mayúscula em la línea no difieren según la prueba de Scott-Knott a $5 \%$ de probabilidad. Los CVs fueron: $C v 1=18,5 \% ; C_{v 2}=17,6$ e $C_{v 3}=19,3 \%$.

Los sustratos arena fina y jiffy ${ }^{\circledR}$ fueron eficientes en la formación y desarrollo del sistema radicular de las miniestacas de G. crinita. En ese sentido, Zietemann y Roberto (2007) indican que la emisión de raíces en mayor número y longitud es fundamental para garantizar la calidad y producción de plantas a nivel comercial. Además, Carvalho Junior et al. (2009) citan que un sistema radicular bien formado tendrá la capacidad de explorar una mayor área de suelo y con ello favorecer la absorción de agua y nutrientes para el mejor desarrollo de la planta en campo definitivo. 


\section{CONCLUSIONES}

Los invernaderos construidos de policarbonato y de malla raschel ${ }^{\circledR}$ asociados con los sustratos arena fina y jiffy ${ }^{\circledR}$ más los aditivos enraizantes rapid $\operatorname{root}^{\circledR}$ y sani ${ }^{\circledR}$ fueron las mejores condiciones para el enraizamiento de miniestacas de G. crinita;

El sistema aeropónico por ser un medio sin sustrato no influenció positivamente el enraizamiento de miniestacas de G. crinita;

Así mismo se recomienda evaluar la sobrevivencia y desarrollo del sistema radicular y parte aérea de las miniestacas enraizadas hasta la formación de plantas aptas para ser trasplantadas a campo definitivo.

\section{AGRADECIMIENTOS}

Los autores agradecen al Consejo Nacional de Ciencia, Tecnología e Innovación Tecnológica (CONCYTEC) através de su unidad ejecutora: Fondo Nacional de Desarrollo Científico, Tecnológico y de Innovación Tecnológica (FONDECYT), por el apoyo financiero para el desarrollo del presente trabajo de investigación. De la misma manera, un agradecimiento especial a los investigadores del instituto de Investigaciones de la Amazonía Peruana-IIAP, por el asesoramiento y acompañamiento "Técnico y Científico" en todas las etapas de la investigación.

\section{REFERENCIAS}

ANGULO, G. R. Influencia del ácido-3-indol butírico y tipo de sustrato en el enraizamiento de Plukenetia huayllabambana en cámaras de sub irrigación en la provincia de San Martín. 95 p. 2016. Thesis (Mestrado en Ingenieria Agronomo) - Universidad Nacional de San Martin, Tarapotp, 2016.

BANDEIRA, F. S.; XAVIER, A.; OTONI, W. C.; LANI E. R. G. Aclimatização ex vitro de plantes propagadas pela enxertia in vitro de clones de Eucalyptus urophylla x E. grandis. Revista Árvore, v. 31, n. 5, p. 773-781, 2007.

BORTOLINI, M. F.; ZUFFELLATO-RIBAS, C.; KOEHLER, H. S.; CARPANEZZI, A. A.; DESCHAMPS, C.; OLIVEIRA, M. C.; BONA, C.; MAYER, J. L. S. Tibouchina sellowiana (Cham.) Cogn: enraizamento, anatomia e analises bioquímicas nas quatro estações do ano. Ciência Florestal, v. 18, n. 2, p. 159-171, 2008.

CARVALHO JÚNIOR, W. G. O.; MELO, M. T. P.; MARTINS, E. R. Comprimento da estaca no desenvolvimento de mudas de alecrim-pimenta. Ciência Rural, v. 39, n. 7, p. 2199-2202, 2009.

CARVALHO, P. E. R. Influência da intensidade luminosa e do substrato no crescimento, no conteúdo de clorofila e na fotossíntese de Cabralea canjerana (Vell.) Mart. Susp. canjerana, Colophyllum brasitiense CAMB. e Cetrolobium robustum (Vell.) Maet. Ex Benth, na fase juvenil. 157 p. Tese (Doutorado em Engenharia Florestal) - Universidade Federal do Paraná, Curitiba, 1996.

CHAGAS, J. H.; PINTO, J. E. B. P; BERTOLUCCI, S. K. V.; NALON, F. H. Produção de mudas de hortelã-japonesa em função da idade e de diferentes tipos de estaca. Ciência Rural, Santa Maria, v. 38, n. 8, p. 2157-2163, 2008.

CUQUEL, F. L.; MINAMI, K. Enraizamento de estacas de crisântemo [Dendranthema morifolium (Ramat.) Tzvelev] tratadas com ácido indolbutirico veiculado em talco. Scientia agrícola, v. 51, n. 1, p. 28-35, 1994.

DI RIENZO, J. A.; CASANOVES, F.; BALZARINI, M. G.; GONZALEZ, L.; TABLADA, M.; ROBLEDO, C. W. InfoStat, versión 2008. Grupo InfoStat, Argentina. 2008. Disponible: <http://www.infostat.com.ar>. Acessado en: 30 jul. 2018.

DIAS, P. C.; OLIVEIRA, L. S.; XAVIER, A.; WENDLING, I. Estaquia e miniestaquia de espécies florestais lenhosas do Brasil. Pesquisa Florestal Brasileira, v. 32, n. 72, p. 453-462, 2012.

DOINA, C.; FIRA, A.; SIMU, M. The role of rooting substrate in blackberry ex vitro rooting and acclimatization stage. Pro Environment, v. 8, p. 280-284, 2015.

ECHEVERRÍA, F.; BARQUERO, M.; RODRÍGUEZ, D. Estudio del desarrollo radical en almácigo de híbridos f1 de CAFÉ obtenidos por cultivo de tejidos. Agronomía Costarricense, v. 38, n. 1, 67-74, 2014

Sci. For., Piracicaba, v. 47, n. 124, p. 632-643, dez. 2019 DOI: doi.org/10.18671/scifor.v47n124.05 
Torres et al. - Enraizamiento de miniestacas de Guazuma crinita M. utilizando

diferentes invernaderos, sustratos y aditivos

FACHinello, J. C.; HOFFMANN, A.; NACHTGAL, J. C.; KERSTEN, E. Propagação de plantas frutíferas. Brasília: Embrapa Informação Tecnológica, 2005.

GATTI, K. C.; SUAREZ, I.; ESPITIA, M.; TOBAR, D. Producción de plántulas por miniestacas de Tectona grandis in Acacia mangium Wild y Gmelina arborea Roxb. Cordoba: Universidad de Cordoba, 2011.

GUERRA, H.; ARÉVALO, L. A.; VÁSQUEZ, A.; GUERRA, W.; DEL CASTILLO, D. Manual técnico de propagación vegetativa de Bolaina blanca (Guazuma crinita Mart.) en ambientes controlados. 2018. Disponible en: < http://repositorio.iiap.org.pe/bitstream/IIAP/378/1/Guerra_documentotecnico_2018.pdf >. Acessado en: 30 jul. 2018

HARTMANN, H. T.; KESTER, D. E.; DAVIES JÚNIOR, F. R.; GENEVE, R. L. Plant propagation: principles and practices. New Jersey: Prentice, 2011.

HARTMANN, H.; KESTER, D.; DAVIES, F.T. Plant Propagation Principles and Practices. New Jersey: PrenticeHall, 1990. 647 p.

HERNÁNDEZ, R.R.J.; OSPINA, P.C.M.; RODAS, P.C.A.; BYRON, U.J.; GODOY, B. J.A.; FABIO ARISTIZÁBAL, V.A. El Eucalipto. Eucalyptus grandis W. Hill ex Maiden: guías silviculturales para el manejo de especies forestales con miras a la producción de madera en la zona andina colombiana. 2006. Disponible:< https:// www.cenicafe.org/es/publications/eucalipto.pdf>. Acessado en: 30 jul 2018.

HUAMÁN, S. E. E. Efecto del área foliar y la frecuencia de riego en el enraizamiento de brotes de Coffea arabica L. "cafeto"; en condiciones controladas. Tesis, Universidad Nacional De La Amazonía Peruana, 2015. Disponible en: < http://repositorio.unapiquitos.edu.pe/bitstream/handle/UNAP/4437/Elias_Tesis_Titulo_2015. pdf? sequence=1\&isAllowed=y>. Acessado en: 30 jul. 2019.

IIAP - INSTITUTO DE INVESTIGACIÓN DE LA AMAZONIA PERUANA. Evaluación Económica de Parcelas de Regeneración Natural y Plantaciones de Bolaina Blanca “Guazuma Crinita”, en el Departamento de Ucayali. 2009. Disponível em: < http://repositorio.iiap.org.pe/bitstream/IIAP/225/1/Alvarez_documentotecnico_2009_11. pdf>. Acesso em: 30 jul 2018.

LANA, R. M. Q.; LANA, A. M. Q.; BARREIRA, S.; MORAIS, T. R.; FARIA, M. V. Doses do ácido indolbutírico no enraizamento e crescimento de estacas de eucalipto (Eucalyptus urophylla). Bioscience Journal, v. 24, n. 3, p. 13-18, 2008.

LEAKEY, R. R. B.; MESÉN, J. F.; TCHOUNDJEU, Z.; LONGMAN, K. A.; DICK, J. M. C. P.; NEWTON, A.; MATIN, A.; GRACE, J.; MUNRO, R. C.; MUTHOKA, P. N. Low technology techniques for the vegetative propagation of tropical trees. The Commonwealth Forestry Review, v. 69, n. 3, p. 247-257, 1990.

LEITE, J. B. V.; MARTINS, A. B. G. Efeito do ácido indolbutirico e época de coleta no enraizamento de estacas semi-lenhosas do cacaueiro. Revista Brasileira de Fruticultura, v. 29, n. 2, p. 204-208, 2007.

MESÉN, F. Enraizamiento de estacas juveniles de especies forestales: usos de propagadores de subirrigación, 1998. 36p. Disponible: < http://repositorio.bibliotecaorton.catie.ac.cr/bitstream/handle/11554/1638/ Enraizamiento_de_estacas.pdf?sequence=1\&isAllowed=y>. Acessado en: 30 jul. 2019.

MURILLO-GAMBOA, O.; BADILLA-VALVERDE, Y.; VILLALOBOS-ARAYA, M.; ROJAS-PARAJELES, F. Optimización de la tecnología de propagación vegetativa in vivo y plantación de teca y pilón. 2013. Disponible en: < https://repositoriotec.tec.ac.cr/bitstream/handle/2238/3243/optimizacion_tecnologia_propagacion_vegetativa_ in_vivo.pdf? sequence=1\&isAllowed=y>. Acessado en: 30 jul 2018.

NICHOLS, M. Aeroponics and potatoes. Acta horticulturae, n. 670, p. 201-206, 2005.

OTAZÚ, V. Manual de producción de semilla de papa de calidad usando aeroponía. Lima: Centro Internacional de la Papa, 2010, 34 p.

PAREDES, O.; SOUDRE, M.; CHÁVEZ, J.; GUERRA, W. Propagación vegetativa de bolaina blanca (Guazuma crinita Mart.) mediante injerto, bajo condiciones ambientales controladas. Folia Amazónica, v. 19, n. 1-2, p. 69-77, 2010.

PIO, R.; CHALFUN, N, N. J.; RAMOS, J, D.; GONTIJO, T, C. A.; TOLEDO, M.; CARRIJO, E, P. Presença de folhas e gema apical no enraizamento de estacas herbáceas de figueira oriundas de desbrota. Revista Brasileira de Agrociência, v. 10, n. 1, p. 51-54, 2004. 
REYNEL, C.; PENNINGTON, R. T.; PENNINGTON, T. D.; FLORES, C.; DAZA, A. Árboles útiles de la amazonía peruana y sus usos: un manual con apuntes de identificación, ecología y propagación de las especies. Lima: Tarea Gráfica Educativa, 2003.

RÍOS, M. Guía para inversionistas interesados en el sector forestal peruano. Lima: AGRIFIN, 2014,132 p.

RITTER, E.; ANGULO, B.; RIGA, P.; HERRÁN, C.; RELLOSO, J.; SAN JOSE, M. Comparison of hydroponic and aeroponic cultivation system for the production of potato minitubers. Potato Research, v. 44, p. 127-135, 2001.

SANTOS, R. G.; SOUSA, I. M.; ALBUQUERQUE, C. C.; SILVA, K. M. B. Tipo de estaca e substrato na propagação vegetativa de Lippia gracilis Schauer. Arquivos do Instituto Biológico, v. 83, p. 1-4, 2016.

SENAMHI. Servicio Nacional de Meteorología e hidrografía del Perú. 2016. Disponible en: < https://www. senamhi.gob.pe/>. Accesado en: 10 jul. 2018.

SINIA-MINAM. Sistema Nacional de información Ambiental. 2016. Disponible en: <http://sinia.minam. gob.pe/indicador/1607>. Accesado en: 10 oct. 2018.

SOUDRE, M.; MUERAS, L.; LIMACHE, A.; GUERRA, H.; MESEN, F.; PEREZ, F. Propagación vegetativa de tornillo Cedrelinga cateniformis (Ducke) mediante enraizamiento de estacas juveniles en propagador de subirrigación. Folia Amazónica, v. 20, n. 1-2, p. 83-94, 2011.

TCHOUNDJEU, Z.; AVANA, M. L.; LEAKEY, R. R. B.; SIMONS, A. J.; ASAAH, E.; DUGUMA, B.; BELL, J. M. Vegetative propagation of Prunus Africana: effects of rooting medium, auxin concentrations and leaf area. Agroforestry System, v. 54, p. 183-192, 2002.

TIMM, C. R. F.; SCHUCH, M. W.; TOMAZ, Z. F. P.; MAYER, N. M. A. Enraizamento de miniestacas a partir de ramos herbáceos de porta-enxertos de pessegueiro, em diferentes substratos. Revista Inova Ciência \& Tecnologia, v. 1, p. 18-22, 2015.

VALVERDE-CERDAS, L.; ALVARADO, L.; HINE, A. Micropropagation of clones from controlled crosses of Gmelina arborea in Costa Rica. New Forests, v. 28, p. 187-194, 2004.

VERNIER, R. M.; CARDOSO, S. B. Influência do ácido indol-butírico no enraizamento de estacas em espécies frutíferas e ornamentais. Revista Eletrônica de Educação e Ciência, v. 3, n. 2, p. 11-16, 2013.

XAVIER, A.; WENDLING, I.; SILVA, R. L. Silvicultura clonal: princípios e técnicas. Viçosa: Editora UFV, 2009. $272 \mathrm{p}$.

YAMAMOTO, L. Y.; BORGES, R. S.; SORACE, M.; RACHID, B. F.; RUAS, J. M. F.; SATO, O.; ASSIS, A. M.; ROBERTO, S. R. Enraizamento de estacas de Psidium guajava L. 'Século XXI' tratadas com ácido indolbutírico veiculado em talco e álcool. Ciência Rural, v. 40, n. 5, p. 1037-1042, 2010.

ZIETEMANN, C.; ROBERTO, S. R. Efeito de diferentes substratos e épocas de coleta no enraizamento de estacas herbáceas de goiabeira, cvs. Paluma e Século XXI. Revista Brasileira de Fruticultura, v. 29, n. 1, p. 31-36, 2007.

Recibido el: 04/01/2019

aceptado en: 17/04/2019

Sci. For., Piracicaba, v. 47, n. 124, p. 632-643, dez. 2019

DOI: doi.org/10.18671/scifor.v47n124.05 